\title{
Growing role for PADs in pathogenesis
}

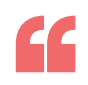

A haplotype

of PADI4, which encodes

PAD4, is a

known risk factor for RA

Peptidylarginine deiminases (PADs) are responsible for the citrullination of many proteins, a posttranslational modification known to be important in the pathogenesis of rheumatoid arthritis (RA). Citrullination of histones is a crucial step in the formation of neutrophil extracellular traps (NETs), but a new study has extended the role of PADs beyond NET formation, or NETosis, to the production of pro-inflammatory cytokines.

Researchers Bo Sun and Nishant Dwivedi, together with their colleagues, characterized a novel relationship between PAD4 and the transcription factor NF- $\kappa \mathrm{B}$. Citrullination of the p65 subunit of NF- $\kappa$ B by PAD 4 increased the binding of this subunit to importin $\alpha 3$ in neutrophils, thereby increasing the rate of nuclear translocation of NF- $\kappa \mathrm{B}$ and the subsequent production of the pro-inflammatory

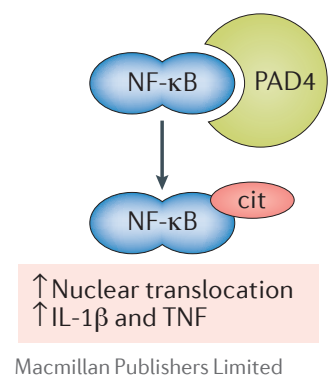

cytokines IL- $1 \beta$ and TNF. "Our data set an example of how citrullination can directly modulate the function of transcription factors and immune cells," says I-Cheng Ho, corresponding author on the study. "By promoting the expression of inflammatory cytokines, such as TNF and IL-1 $\beta$, hypercitrullination can also actively contribute to the pathogenesis of RA," he continues.

A haplotype of PADI4, which encodes PAD4, is a known risk factor for RA; this risk haplotype contains three missense mutations located away from the site of enzymatic activity. The researchers showed that these missense mutations increased the affinity of the resulting PAD4 variant for NF- $\kappa$ B $p 65$ compared with normal PAD4. This increased affinity led to enhanced citrullination, nuclear translocation and expression of pro-inflammatory cytokines in vitro, providing a possible explanation for the increased risk of RA in carriers of this gene variant.

Using mass spectrometry and computer modelling, the researchers identified four arginine residues on NF- $\kappa B$ p 65 that they believe are crucial for the increased activity observed after citrullination by PAD4. "In the near future, we plan to characterize in detail the dynamic interaction between PAD4 and NF- $\kappa$ B p 65 and understand how citrullination of p65 enhances its interaction with importins," reports Ho.

"The paper opens the door for many interesting new studies, both on basic mechanisms and on therapies using various PAD inhibitors," states Lars Klareskog, who was not involved in this study. "Specifically targeting the interaction between PAD4 and NF- $\kappa$ B p65 may allow us to inhibit the activity of NF- $\mathrm{kB}$ without the unwanted effects of global inhibition of PAD activity," explains Ho.

"The role of citrullination in neutrophil activation was already known and believed to be of importance in RA, in particular in relation to the influence of ACPAs on NETosis, and the present findings add significantly to the understanding of the potential role of neutrophils in RA and other inflammatory diseases," says Klareskog. "In addition, the findings identify a new and interesting and cell specific potential target for therapy," he concludes.

Joanna Collison

ORIGINAL ARTICLE Sun, B. et al. Citrullination of $\mathrm{NF}-\mathrm{kB}$ p 65 promotes its nuclear localization and TLR-induced expression of IL-1 $\beta$ and TNF $\alpha$. Sci. Immunol. 2, eaal3062 (2017) 